\title{
Avaliação de patologia psiquiátrica pelo instrumento ChA- PAS (versão para crianças e adolescentes da PAS-ADD) numa população de crianças e jovens com deficiência intelectual grave e profunda
}

\author{
JOÃO ALBUQUERQUE* \\ Centro de Recuperação de Menores D. Manuel Trindade Salgueiro; Instituto das Irmãs Hospitaleiras do Sagrado \\ Coração de Jesus
}

\begin{abstract}
Resumo: A Deficiência Mental, aqui designada por Perturbação de Deficiência Inteletual (PDI), de acordo com o grupo de trabalho da CID 11, apresenta grande prevalência de morbilidade psiquiátrica associada, insuficientemente diagnosticada. A perspectiva do Diagnóstico Duplo na PDI procura clarificar esta realidade no quadro global das dificuldades apresentadas pela pessoa com PDI. Este artigo apresenta os dados resultantes da aplicação de um instrumento desenhado para a pesquisa de patologia psiquiátrica em crianças e jovens com PDI, a ChA-PAS (Child Adolescent Psychiatric Assessment Schedules), e avalia a consistência dos resultados deste com os diagnósticos psiquiátricos previamente realizados clinicamente.

Nos resultados, a grande maioria da população estudada (91\%) apresenta patologia psiquiátrica associada à PDI, com predomínio da Perturbação do Espectro do Autismo (PEA) e a Perturbação de Hiperactividade e Défice de Atenção (PHDA). A patologia prevalecente nas pessoas com PDI profunda é a PEA, enquanto nas pessoas com PDI Grave a PHDA é a mais representada. O grau de concordância com os diagnósticos clínicos prévios foi de $100 \%$, o que apoia a hipótese de a ChA-PAS ser um instrumento com interesse na avaliação desta população.

Concluímos que mesmo com instrumentos adaptados, a avaliação de pessoas com PDI, sobretudo de nível Grave e Profundo, é sempre um desafio. A exploração de novos instrumentos e a sua tradução, aferição, e validação para a população portuguesa poderá ser uma mais-valia para as pessoas com PDI.
\end{abstract}

Palavras-chave: Deficiência Intelectual; Diagnóstico Duplo; Instrumentos de Avaliação.

\section{Introdução}

A Perturbação do Desenvolvimento Intelectual (PDI), designação considerada pelo grupo de trabalho da CID-11 para a Deficiência Mental (CID-10), é uma entidade que percorre transversalmente todas as áreas de intervenção em reabilitação. Esta realidade tem sido, desde sempre, fonte de graves limitações no apoio a estas pessoas, pela dificuldade de articular todas as áreas que deveriam estar presentes simultaneamente no plano de intervenção.

Nomeadamente, na área da psiquiatria e saúde mental, não existe uma cultura que valorize a especificidade e as dificuldades da abordagem destas situações. Não há por isso formação específica sobre o tema dentro do currículo dos médicos do internato complementar de psiquiatria e saúde mental, quer de adultos, quer de infância e adolescência, perpetuando um modelo de apoio que ra-

*Correspondência: j.p.alb@hotmail.com ramente preenche as necessidades das pessoas com PDI, das suas famílias, ou dos técnicos que as acompanham em instituições de reabilitação, habitualmente ligadas ao sector social.

Dentro destas especificidades, destaca-se a abordagem na perspectiva do Diagnóstico Duplo ${ }^{1}$, que procura clarificar, no quadro global das dificuldades apresentadas pela pessoa com PDI, o que é resultante da PDI, e o que se associa a diagnósticos psiquiátricos, que, pelo exposto anteriormente, muitas vezes não são reconhecidos, e são antes englobados no quadro da PDI.

Consideramos que é importante produzir evidência científica que desperte a comunidade psiquiátrica para a realidade do Diagnóstico Duplo, quer como forma de melhorar a intervenção do psiquiatra nestes quadros, quer também como forma de sensibilizar os responsáveis pela formação médica para a necessidade de integrar esta área nos currículos das especialidades. 
A maior prevalência de doença mental nas pessoas com PDI é um facto amplamente reconhecido. Infelizmente, existem poucos instrumentos desenhados para aplicação nesta população, e os que existem têm habitualmente limitações significativas nas populações abrangidas (idade, grau de deficiência), nas possibilidades de recolha de informação, e na quantidade de diagnósticos que permitem.

\section{Objectivo}

Este artigo apresenta os dados resultantes da aplicação de um instrumento desenhado para a pesquisa de patologia psiquiátrica em crianças e jovens com PDI, a ChA-PAS (Child Adolescent Psychiatric Assessment Schedules), e avalia a consistência dos resultados deste com os diagnósticos psiquiátricos previamente realizados clinicamente.

Os dados apresentados correspondem à parte da avaliação psiquiátrica de um estudo mais abrangente em curso no Centro de Recuperação de Menores D. Manuel Trindade Salgueiro (CRM), das Irmãs Hospitaleiras do Sagrado Coração de Jesus (IHSCJ), o Estudo Multidimensional da População Infantil e Juvenil com DI Grave e Profunda do CRM, que tem como principal objectivo, entre outros, criar uma bateria de avaliação multidisciplinar que satisfaça as necessidades das diversas áreas técnicas relativamente à intervenção com crianças e jovens com PDI Grave e Profunda. Futuramente, serão também publicados os dados relativos ao estudo global.

O CRM assiste 120 residentes do sexo feminino e a idade de acolhimento está compreendida entre os 3 e os 17 anos. É condição necessária apresentarem o diagnóstico de Perturbação do Desenvolvimento Intelectual. Actualmente o CRM privilegia situações de Diagnóstico Duplo (PDI e outras perturbações no âmbito da saúde mental e psiquiatria) e de Multideficiência (PDI e outras deficiências sensoriais, expressivas, ou motoras), como área clínica de intervenção mais específica da sua prática. Tem um âmbito geográfico de intervenção nacional.

A sua especificidade surge das características do quadro técnico, que engloba, ao contrário do que é habitual nas instituições ligadas à reabilitação, pessoal médico e de enfermagem. Integra uma psiquiatra, uma médica de medicina geral e familiar, uma pediatra, uma médica de medicina dentária, 14 enfermeiros, uma psicóloga, uma técnica superior de serviço social, uma terapeuta ocupacional, uma psicomotricista, uma fisioterapeuta, uma terapeuta da fala, duas educadoras sociais, 52 cuidadoras, quatro com funções específicas de acompanhamento de actividades ocupacionais, duas professoras de ensino especial, e seis irmãs da congregação das IHSCJ, com actividades de direcção e coordenação de serviços, que acumulam com serviço assistencial. A "ChA-PAS"pertence a um conjunto de quatro instrumentos globalmente designados PAS-ADD (Psychiatric Assessment Schedules for Adults With Developmental Disabilities) (http://www.pas-add.com/) destinados a me- lhorar a detecção e diagnóstico de problemas de saúde mental em pessoas com PDI. A PAS-ADD Clinical Interview é uma entrevista clínica semi-estruturada resultante da evoluçãoda versão inicial PAS-ADD 10, destinada ao uso por profissionais treinados, tipicamente psiquiatras ou psicólogos, e que permite diagnósticos precisos dentro dos principais diagnósticos da CID-10 e DSM-IV-TR. A PAS-ADD Checklist é um questionário de 25 itens, escrito em linguagem comum, desenhado a pensar no uso primordial por cuidadores e familiares, que são as pessoas que mais rapidamente dão conta de alguma alteração no comportamento das pessoas que cuidam, com o objectivo de decidirem da necessidade de uma avaliação em contexto clínico. A Mini PAS-ADD é um questionário semi-estruturado destinado à detecção de sintomas psiquiátricos, conduzido por um elemento da equipa, que não tendo de ter formação psiquiátrica ou psicológica, tem no entanto de receber o treino apropriado para o usar. É habitualmente aplicado aos cuidadores mais directos ou a familiares da pessoa com PDI. Finalmente, a ChA-PAS é o elemento mais recente da família PAS-ADD, e resultou de inúmeras solicitações de profissionais, pelo limite mínimo de idade de aplicação da Mini PAS-ADD. É também destinada à utilização por pessoal da equipa que tenha recebido o treino adequado, podendo ser entrevistados quer a criança ou jovem com PDI, quer um cuidador ou familiar, ou todos.

À excepção da PAS-ADD Checklist, nenhum destes instrumentos se destina a determinar um diagnóstico, mas permite perceber com grande precisão a necessidade de avaliação clínica para definir a presença de alguma perturbação. Tem a grande vantagem de potenciar pessoal sem treino em psiquiatria ou psicologia para a detecção de eventual doença psiquiátrica, alargando muito as possibilidades de avaliação da população com PDI, em diferentes contextos e em todas as idades e graus de deficiência.

A ChA-PAS é um instrumento de 97 itens, organizados em sete secções, associados a sintomas psiquiátricos, quantificados numa escala de gravidade de dois ou quatro pontos. Os itens são baseados na CID-10 (OMS, 1992). Alguns itens referem-se a sintomas nucleares de patologias específicas, enquanto outros são comuns a várias patologias. A ChA-PAS gera assim pontuação em nove escalas de sintomas: Psicose, Episódio Maníaco, Episódio Depressivo, Perturbação Ansiosa, Perturbação Obsessivo-Compulsiva, Perturbação da Conduta, Perturbação de Hiperactividade e Défice de Atenção (PHDA), Perturbação do Espectro do Autismo (PEA). Cada escala apresenta uma pontuação de referência para identificar a presença provável do respectivo problema de saúde mental

\section{Epidemiologia}

A PDI apresenta uma prevalência, segundo vários estudos ${ }^{2}$ citados por Albuquerque $(2000)^{3}$ de cerca de $1 \%$, quando 
utilizados pelo menos dois critérios de avaliação, o quociente de inteligência (QI) e uma escala de comportamento adaptativo. Este valor difere significativamente do que encontramos se utilizarmos apenas o QI, que é de 3\%.Tal explica também a variação de resultados que se verifica entre os diversos estudos. A prevalência da PDI Grave e Profunda é muito inferior à da PDI Ligeira e Moderada $(0,4 \%$ face a 2,5 a $3 \%)$, e as suas causas são habitualmente mais definidas ${ }^{4}$.

Muito mais escassos são os estudos de prevalência de doença mental na PDI. No entanto, todos os estudos existentes concordam na ideia de que as pessoas com PDI têm uma vulnerabilidade aumentada para problemas de saúde mental ${ }^{5-7}$. Encontra-se uma prevalência de $30 \%$ a $40 \%$ de doença mental nas pessoas com PDI, o que corresponde a cerca do quádruplo da prevalência na população geral. Esta relação mantém-se para cada patologia específica que consideremos. Assim, para a Perturbações Esquizofrénicas a prevalência é de $3 \%{ }^{8}$, e as Perturbações Ansiosas ocorrem em cerca de 40\% das pessoas com PDI (Raghavan, 1997) ${ }^{9}$. Exceptuam-se as Perturbações do Humor, com prevalências inferiores ou semelhantes à população geral nos poucos estudos realizados entre 1,3 e $3,7 \%$ para a Depressão ${ }^{10}$, $6,6 \%$ para todas as perturbações do humor ${ }^{5}$.

\section{Método}

A amostra corresponde ao universo total das residentes no CRM com idades compreendidas entre os três e os 25 anos de idade, e portadoras de PDI Grave e Profunda.

A aplicação da ChA-PAS insere-se no Estudo Multidimensional em curso no CRM, com já dissemos, e diz respeito à pesquisa de patologia psiquiátrica. Este instrumento considera, na sua aplicação, um informador principal, que pode entrevistar quer a pessoa a quem se aplica a escala, quer outras pessoas que contactem regularmente com ela (família, outros cuidadores), e um segundo informador, que acompanhe a pessoa selecionada noutros contextos (habitualmente o professor, na escola).

Porque não há versões traduzidas e validadas para a população portuguesa, optámos por utilizar a versão original, com o cuidado de ser sempre o mesmo informador a utilizar a escala. Considerámos que, quer uma tradução livre da nossa parte, quer a utilização de outras versões já traduzidas (espanhol, francês, etc.) poderiam afectar mais significativamente os resultados.

Assim, escolhemos o médico psiquiatra do CRM como primeiro informador para cada pessoa selecionada, por ser o elemento da equipa mais directamente ligado à patologia psiquiátrica, o que lhe permitia a utilização da escala sem necessitar de formação específica. Acresce o facto de conhecer todas as pessoas selecionadas, e ter acesso a todos os outros cuidadores. Como segundo informador, era pedido ao técnico de referência da pessoa selecionada que se responsabilizasse por esse papel, por possuir conheci- mento privilegiado da pessoa, e por colectar regularmente informação de outros informadores.

Foram utilizadas todas as possibilidades de colheita de dados, desde a entrevista com a pessoa selecionada, de acordo com a possibilidade de participação desta, até à participação de outros cuidadores ligados a actividades básicas de subsistência, outras intervenções técnicas, e à área pedagógica. Foram considerados todos os dados disponíveis ao longo da história da pessoa selecionada, já que muitas das alterações consideradas não estão presentes no momento da entrevista. Aliás, a referência ao tempo considerado para a colheita de dados é um dos aspectos que a ChA-PAS obriga a indicar no início do preenchimento.

A caracterização da pessoa selecionada foi baseada nos dados presentes no processo clínico de cada uma (dados biográficos e diagnósticos prévios). O estudo abarcou toda a população do CRM com DI Grave e Profunda, dentro da idade definida.

\section{Resultados \\ Amostra}

Um total de 33 residentes do sexo feminino do CRM foram avaliadas, com idade entre os três e os 25 anos. Destas, 23 apresentavam PDI Grave e 10 PDI Profunda.

Dos dados clínicos prévios que constam do processo clínico de cada residente avaliada, verificou-se que $30(90,9 \%)$ delas tinham um diagnóstico psiquiátrico, $28(84,8 \%)$ tinham patologia somática, $31(93,9 \%)$ apresentavam duas ou mais patologias associadas, com destaque para a Epilepsia, presente em 10 (33,3\%), e, finalmente, não existia nenhuma residente avaliada sem qualquer patologia associada.

Quadro 1: Caracterização da amostrade acordo com os dados do processo clínico

\begin{tabular}{|l|c|c|c|}
\hline $\begin{array}{c}\text { Perturbação } \\
\text { Desenvolvimento } \\
\text { Intelectual }\end{array}$ & $\begin{array}{c}\text { Grave } \\
\mathbf{( N = 2 3 )}\end{array}$ & $\begin{array}{c}\text { Profundo } \\
\mathbf{( N = 1 0 )}\end{array}$ & $\begin{array}{c}\text { Total } \\
\mathbf{( N = 3 3 )}\end{array}$ \\
\hline Género & Feminino & Feminino & \\
\hline Idade 3<6 & 2 & 1 & 1 \\
\hline Idade 6<12 & 19 & 8 & 5 \\
\hline Idade 12<25 & 20 & 9 & 29 \\
\hline Perturbação Psiquiátrica & 16 & 13 & 29 \\
\hline Perturbação Somática & 16 & 9 & 25 \\
\hline $\begin{array}{l}\text { Com Perturbação } \\
\text { Psiquiátrica e Somática }\end{array}$ & 0 & 0 & 0 \\
\hline Sem outros diagnósticos & 0 & & \\
\hline
\end{tabular}

\section{Aplicação da CHA-PAS}

Após a aplicação do instrumento ChA-PAS, verificámos uma predominância de cotação acima da pontuação de referência para os diagnósticos de Perturbação do Espectro do Autismo (PEA) ( $\mathrm{n}=13$, ou 43,3\%), e de Pertur- 
bação de Hiperactividade e Défice de Atenção (PHDA) ( $n=11$, ou $36,6 \%$ ), o que está de acordo com a experiência dos técnicos que trabalham nesta área e com os dados da literatura.

Vários casos apresentam cotações abaixo do limiar significante (definido pela escala no documento de registo de dados) para Perturbação da Conduta, mas todos eles têm cotação significativa para PHDA, diagnóstico que integra as alterações de conduta identificadas. Outros apresentam cotações sub-liminares para psicose e perturbação obsessiva, mas todos pertencem também aos casos que pontuam significativamente para PEA, que integra os sintomas psicóticos e obsessivos identificados.

Finalmente, embora só duas pessoas pontuem para Perturbação Ansiosa (PA), existem mais 11 que, embora pontuem significativamente para PA, cotam também para PHDA e PEA. Estes são diagnósticos mais específicos, que incluem as alterações de ansiedade, inespecíficas e presentes em muitas patologias, e que por isso não podem ser cotadas como diagnóstico isolado nestes casos.

Tabela 2: Resultados da aplicação da ChA-PAS

\begin{tabular}{|c|c|c|c|c|}
\hline $\begin{array}{l}\text { Perturbação do Desenvolvimento } \\
\text { Intelectual (n:33) }\end{array}$ & Grave & Profundo & Total & Notas \\
\hline PEA & $8(26,6 \%)$ & $5(16,6 \%)$ & $13(43,3 \%)$ & \\
\hline PHDA & $10(33,3 \%)$ & $1(3,3 \%)$ & $11(36,6 \%)$ & \\
\hline Episódio Maníaco & $5(16,6 \%)$ & 0 & $5(16,6 \%)$ & \\
\hline Episódio Depressivo & $2(6,6 \%)$ & $1(3,3 \%)$ & $3(9,9 \%)$ & \\
\hline Perturbação Ansiosa & $2(6,6 \%)$ & 0 & $1(3,3 \%)$ & $\begin{array}{l}\text { Mais } 11(36,6 \%) \text { pontuam, } \\
\text { integradas em PEA e PHDA }\end{array}$ \\
\hline Perturbação da Conduta & $1(3,3 \%)$ & 0 & $1(3,3 \%)$ & \\
\hline Sem diagnóstico Psiquiátrico & $1(3,3 \%)$ & $2(6,6 \%)$ & $3(9,9 \%)$ & \\
\hline
\end{tabular}

Gráfico 1: Resultados da aplicação da ChA-PAS

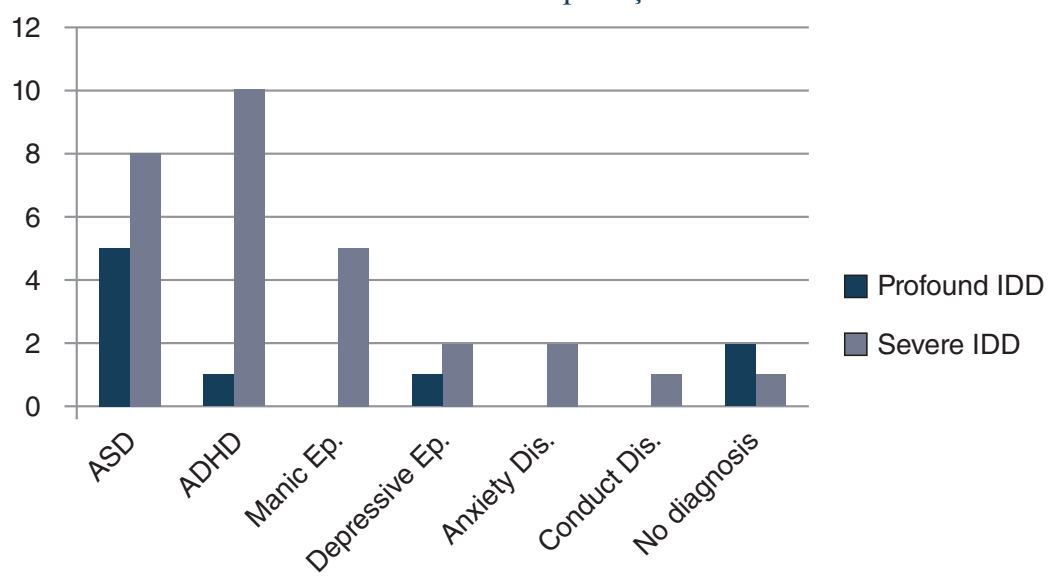

\section{Discussão e Conclusões}

O presente trabalho procura demonstrar a importância do instrumento ChA-PAS na detecção de provável patologia psiquiátrica em crianças e jovens com PDI Grave e Profunda. Sendo a colheita de informação cega em relação aos diagnósticos anteriores, o grau de concordância com os diagnósticos clínicos prévios foi de $100 \%$, o que apoia a hipótese de a ChA-PAS ser um instrumento com interesse na avaliação desta população.

Algumas das doentes estudadas apresentam patologia inespecífica e complexa, em que o quadro sindromático engloba sintomas comuns a várias patologias associadas a alterações do comportamento. Verificámos que a ChA-PAS permitiu identificar o diagnóstico mais estruturante do conjunto de sintomas apresentados, com implicações significativas na orientação terapêutica da doente. O trabalho realizado permitiu-nos também identificar algumas limitações que a avaliação desta população encerra. Assim, foi evidente a dificuldade de avaliar os sintomas de natureza cognitiva (p.ex., ideias de suicídio, auto-confiança, ideias delirantes), pelos problemas cognitivos que as pessoas avaliadas apresentaram. Também a expressão pessoal limita a participação da pessoa no estudo, seja a ausência de expressão verbal, seja o estar acamada, ou mesmo não ter 
qualquer possibilidade de expressão (mesmo a expressão facial está comprometida ou é estereotipada).

A par das dificuldades expressivas e conceptuais, também as dificuldades sensoriais, nomeadamente amaurose bilateral e surdez completa, comprometem significativamente a comunicação eficaz com os avaliadores.

Em alguns itens, nomeadamente sono e apetite, a medicação e as rotinas da instituição modelam-nos, sendo fundamental socorrermo-nos dos dados da história da pessoa avaliada para tentar perceber qual o funcionamento natural desta.

Finalmente, o facto de o primeiro informador ser o psiquiatra que acompanha as pessoas avaliadas, deverá ser tido em conta como factor significativo nos resultados, mesmo que este não tivesse presente no momento da avaliação dos diagnósticos prévios das pessoas avaliadas.

Podemos concluir, ainda assim e tendo presentes as limitações referidas, que a grande maioria da população estudada (91\%) apresenta patologia psiquiátrica associada à $\mathrm{PDI}$, com predomínio da PEA e a PHDA. A patologia prevalecente nas pessoas com PDI profunda é a PEA, enquanto nas pessoas com PDI Grave a PHDA é a mais representada.

A prevalência de patologia psiquiátrica associada à PDI é muito superior na população estudada relativamente aos dados da literatura (91\% para $30 \%$ a $40 \%$ ). Tal pode ser explicado pelo contexto institucional em que decorreu o estudo. Como dissemos antes, o CRM acolhe preferencialmente pessoas com Diagnóstico Duplo, em que a PDI se associa a outro diagnóstico psiquiátrico, tendo por isso uma população em que esta realidade é muito mais comum.

O facto de existir uma menor variedade de diagnósticos psiquiátricos na população com PDI Profunda está de acordo com os dados da literatura. No entanto, não podemos deixar de o questionar face às limitações referidas, nomeadamente no que respeita às capacidades expressivas das pessoas observadas, tanto mais que este facto é mais relevante nas pessoas com multideficiência, em que essas limitações são mais evidentes.

\section{Orientações Futuras}

Mesmo com instrumentos adaptados, a avaliação de pessoas com PDI, sobretudo de nível Grave e Profundo, é sempre um desafio. São por isso necessários mais estudos utilizando este ou outro instrumento construído para a detecção de provável patologia psiquiátrica em pessoas com PDI, para confirmar os dados do presente estudo, para que mais técnicos ganhem experiência e segurança na utilização destes instrumentos, e para alargar a evidência desta realidade.

A exploração de novos instrumentos e a sua tradução, aferição, e validação para a população portuguesa poderá ser uma mais-valia inestimável para as pessoas com PDI, ao ajudar todos os que trabalham com elas a detectar a presença de patologia psiquiátrica concomitante, independentemente da sua formação de base que possuam.

A sensibilização e formação dos profissionais de saúde para a realidade do Diagnóstico Duplo na PDI é fundamental, dada a dimensão desta realidade. Ainda hoje continua a não existir uma formação estruturada sobre a PDI no percurso formativo da classe médica em Portugal, seja a nível pré ou pós-graduado. Só com a produção de evidência científica desta realidade teremos argumentos para apoiar mudanças curriculares que contemplem também esta temática.

\section{Agradecimentos}

Este estudo teve o apoio financeiro do Plano Nacional de Saúde Mental (PNSM) - Direcção Geral da Saúde (DGS) Ministério da Saúde, a quem agradecemos este apoio e todo o estímulo para desenvolver este trabalho. A equipa técnica do CRM agradece a toda a comunidade do CRM, nomeadamente às residentes e seus familiares, o apoio que deram em todo o momento para a realização deste estudo.

\section{Conflitos de Interesses}

Este trabalho é apoiado pelo Plano Nacional de Saúde Mental - Direcção Geral de Saúde, comprometendo os autores para a conclusão do mesmo. Não existem conflitos de interesses dos autores com qualquer outra entidade.

\section{Bibliografia}

1. Carvalho A. Encontro de Trabalho Saúde Mental na Deficiência Intelectual. Comunicação na Fundação Calouste Gulbenkian, Lisboa, 2012.

2. Mercer, J. Labeling the mentally retarded: Clinical and social system perspective on mental retardation. University of California Press, 1973.

3. Albuquerque, MC. A criança deficiente mental ligeira: apectos comportamentais e familiares. INR, Lisboa, 2000.

4. Tejada MI. Retraso mental de origen genético. Presentación de la Red GIRMOGEN, Rev Neurl. 2006; 42 (Supl 1): S1-6.
5. Cooper SA, Smiley E, Morrison J, Williamson A, Allen L. Mental Ill-Health in Adults With Intellectual Disabilities: Prevalence and Associated Factors. British Journal of Psychiatry. 2007; 190: 27-35.

6. Moss S. Psychiatric Disorders in Adults with Mental Retardation, International Review of Research in Mental Retardation. 2002; 24: 211-43.

7. Whitaker S, Read S. The Prevalence of Psychiatric Disorders among People With Intellectual Disabilities: an Analysis of the Literature. Journal of Applied Research inIntellectual Disabilities. 2006; 19: 330-45. 
8. O'Hara J, Sperlinger A. Adults with Learning Disabilities: A PraticalAproach for Health Professionals. Wiley, 1998.

9. Raghavan, R, Anxiety disorders in People with Learning Disabilities: A review o of the literature. Journal of Learning Disabilities for Nursing, Health and Social Care. 1997; 2(1): 3-9.
10. Deb S, Thomas M. Mental disorder in adults with intellectual disability: prevalence of functional psychiatric illness among a community-based population aged between 16 and 64 years". Journal of Intellectual Disability Research. 2001; 45: 495505. 